\title{
Manejo De Miedo, Estrategias De Afrontamiento $Y$ Cultura Ciudadana Para Prevención De La Violencia
}

\section{Sarah Margarita Chávez, Valdez, PhD}

Escuela Libre de Psicología, A.C.,

Chihuahua ELPAC (Escuela Libre de Psicología, A.C.) Universidad de Ciencias del Comportamiento, Departamento de Comunicación Social, Calle Camino a Universidad La Salle Núm. 8805, Col. Labor de Terrazas, 31205, Chihuahua, Chih., México.

\section{Oscar Armando Esparza del Villar, PhD}

Universidad Autónoma de Ciudad Juárez

Av. Universidad y Heroico Colegio Militar 3775, Col. Foviste Chamizal, 32300, Cd. Juárez, Chih., México

Doi: 10.19044/esj.2018.v14n5p133 URL:http://dx.doi.org/10.19044/esj.2018.v14n5p133

\begin{abstract}
In this study, an intervention program was designed and evaluated. This paper focuses on identifying beliefs, perceptions, and negative attitudes in the group, in order to continue with the promotion of positive socialemotional coping and the reduction of social fear. This, however, would help to elevate the behaviors of citizen participation and to inhibit antisocial behaviors. A sample of 47 women and 5 men, a total of 52 students from 17 to 21 years, were trained by means of didactic skill of cases and training in conflict management for a period of 11 weeks in a weekend modality. A quasiexperiment was designed using repeated measures, pretest-interventionpostest, and a control group. As a result, an increase in culture and citizen participation was found in the treatment group after 44 hours of intervention. Qualitative data indicate findings regarding group cognitive relations, assertive coping strategies in conflicting social situations, ability to analyze negative feelings, and a decrease in antisocial behavior.
\end{abstract}

Keywords: Coping strategies, collective fear, posttraumatic stress traits, civic culture

\section{Resumen}

Se plantea y evalúa un programa de intervención con el fín de disminuir el miedo social y estrategias fisio afectivas, promoviendo un afrontamiento proactivo en el ámbito cognitivo conductual, como opción a la 
anti sociabilidad, falta de cultura cívica y efectividad social en colectivos juveniles, analizando: sentimientos, percepciones, sensaciones y conductas negativas, mediante pruebas de auto informe, con diversas técnicas dirigidas y auto reguladas grupalmente. La muestra consistió de 47 mujeres y 5 hombres, un total de 52 jovenes, en rango etario de 17 a 21 años. El tratamiento se manejó mediante un taller avocado a la identificación de factores de riesgo y promoción de estrategias de afrontamiento proactivas. Para este efecto, se trabajó con un cuasi-experimento utilizando medidas repetidas, pretestintervención-postest, con grupo control. Se implementó en modalidad sabatina, resultando en 44 horas de intervención. Los resultados muestran: diferencias en puntuaciones de medias para 9 de 11 de las variables de estudio pre y post-tratamiento, en aspectos pro sociales de la cultura ciudadana y, en los factores de riesgo, entre otros, en los procesamientos cognitivos y conductuales negativos y, respuestas fisio-afectivas que configuraban rasgos de estrés postraumático en los jóvenes.

Palabras-Claves: Afrontamiento, miedo colectivo, estrés post traumático, cultura ciudadana

\section{Introducción}

La conceptualización de juventud es relativa al contexto, se estructura en sociedad, el ser joven cambia de forma y contenido en espacio y tiempo, por ende, los "valores asociados a este grupo de edad y los ritos que marcan sus límites, explican la dependencia infantil y la autonomía adulta" (Feixa, 1998, p.3), los adolescentes atraviesan por diversos procesos de aprendizaje en su iniciación como adultos, que definen y consolidan su identidad biopsicosocial en la etapa adulta.

El joven, dadas sus condiciones y estilo de vida, está en contacto permanente con situaciones de riesgo (Gómez, 2008), esto les hace proclives a participar en hechos y ambientes de violencia. Además, existen factores precipitadores de las conductas antisociales, tal es el caso de: ansiedad, depresión y frustración (Santos, Villa, García, León, Quezada, y Tapia, 2003).

Distintas aproximaciones, fundamentalmente teóricas, proponen multitud de tipologías de la agresión. Aunque de criterios muy diferentes, la mayoría muestran cierta consistencia respecto a la intencionalidad común del agresor -por qué agrede-, y si aquella motivación primaria es la de provocar dolor o daño en la víctima.

Existen tipologías específicas de conductas agresivas relativas a la frustración: la positiva/constructiva, que sería adaptable y prosocial, y otra negativa/destructiva, que sería desadaptativa y antisocial (Lanford, Dodge, Bates, Petit , 2002). 
Dammert y Malone (2006), encontraron que: las carencias estructurales e inseguridades de carácter económico, salud, alimentaria, ambiental y política, eran la principal variable explicativa del miedo al atraco o asalto. En esta misma línea, la victimización sufrida se asocia a una alza en la percepción de riesgo, ambos objeto de atención en las investigaciones del miedo al crimen y constituyen un tema central en la presente intervención, además de los procesos y mecanismos psicosociales asociados al miedo colectivo (Villareal y Silva, 2006; Dammert y Malone, 2006; Vozmediano y San Juan, 2006).

En este orden de cosas, los estudios abarcan temas como la participación comunitaria, satisfacción vecinal, movilidad espacial, redes sociales, cohesión, así como: el grado de tolerancia social hacia la delincuencia, en colonias con altos niveles de desorganización y daño psico social, infiltradas por el narcotráfico.

De acuerdo a ello, la prevención del crimen, podría basarse en fomentar aquellos factores que mejoren la eficacia colectiva en zonas de alta infiltración y riesgo psicosocial. También, cabría esperarse que la participación ciudadana incida en la percepción de seguridad, puesto que si aquella conduce a una disminución de la criminalidad, las comunidades permanecerán unidas y proactivas hacia el alcance de un estilo de vida multiplicador del cambio social, con estilos de vida y proyectos comunitarios preventivos y saludables.

El miedo al delito resulta un área de interés para la criminología, las ciencias sociales y de la conducta, en específico para la psicología social, por su incidencia en hábitos de auto-protección y regulación ciudadana en contextos vulnerados por la violencia (Niño, Lugo, Rozo y Peña, 1998; Peña, 2005), a su vez, resulta crucial la regulación de los niveles de percepción de amenaza, ya que: favorecen altos niveles de temor criminal y por consecuencia, promueven colectivos ineficaces, provoca un desgaste emocional y físico en los individuos, un desajuste en el gasto e inversión privada y pública en la contención del delito $\mathrm{y}$, procesos de discriminación entre grupos, específicamente en los regímenes políticos democráticos (Dammert y Malone, 2006).

En torno a salud pública y victimización, desde una perspectiva psicosocial, identificamos al miedo al crimen como un sentimiento de ansiedad social difusa que afecta a las juventudes y a la percepción de riesgo como un factor ambivalente, que en sociedades sanas incide en hábitos de protección y auto cuidado, pero que, en ambientes de alta violencia social confunde y desgasta el bienestar y calidad de vida de los individuos; Así mismo, el tejido social que los rodea. 


\section{Planteamiento del problema}

Se estima que el nivel de afectación en las puntuaciones de las medias en las estrategias de afrontamiento considerando los siguientes componentes estratégicos: afectivo, fisiológico, cognitivo y conductual es alto de manera negativa, y existe baja participación, eficacia colectiva y cultura ciudadana en los jovenes aunada al temor emocional al crimen y percepción de riesgo.

\section{Método}

Objetivo

El objetivo general del estudio se avoca a promover el desarrollo del grupo, identificar y analizar creencias-percepciones-actitudes negativas, promover estrategias de afrontamiento proactivas, disminución del miedo social, y con ello, incidir en el aspecto fisio afectivo relacionado al desgaste emocional y percibido del miedo, mediante una intervención colectiva.

\section{Viabilidad}

La manipulación y el control de las variables permitió una flexibilidad, eficiencia y manipulación estadística. El proyecto de intervención, fue un proceso que implicó la identificación, diseño, implementación y evaluación de alternativas de respuesta de carácter innovador, con el fin de obtener respuesta a factores de riesgo especificados en el objetivo general y aquellos pro sociales, como respuesta a una necesidad social incidiendo en colectivos juveniles.

\section{Hipótesis}

Hil: Existen diferencias significativas en los puntajes de las medias en las estrategias de afrontamiento afectivas, fisiológicas, cognitivas y conductuales: a nivel personal, familiar y comunitario, en torno al miedo emocional al crimen o miedo difuso y la percepción de riesgo intra sujetos, después de la intervención en el grupo tratamiento pre y post prueba.

Hi2: Existen diferencias significativas en los puntajes de medias en las estrategias de afrontamiento afectivas, fisiológicas, cognitivas y conductuales: a nivel personal, familiar y comunitario, en torno al miedo emocional al crimen o miedo difuso, y la percepción de riesgo inter sujetos, entre el grupo tratamiento y los jóvenes del grupo control. Hol: No Existen diferencias significativas en los puntajes de las medias en las estrategias de afrontamiento afectivas, fisiológicas, cognitivas y conductuales: a nivel personal, familiar y comunitario, en torno al miedo emocional al crimen o miedo difuso y la percepción de riesgo intra sujetos, después de la intervención en el grupo tratamiento pre y post prueba. 
Ho2: No existen diferencias significativas en los puntajes de las medias en las estrategias de afrontamiento afectivas, fisiológicas, cognitivas y conductuales: a nivel personal, familiar y comunitario, en torno al miedo emocional al crimen o miedo difuso, y la percepción de riesgo inter sujetos, entre el grupo tratamiento y grupo control.

\section{Nivel de significación}

Para todo valor de probabilidad igual o menor que 0.05, en el grupo tratamiento, se aceptan las Hipótesis de Investigación $H i(1,2)$ y se rechaza $\mathrm{Ho}(1,2)$.

La intervención consistió en el diseño e implementación de un taller semanal durante los cursos escolares, en una universidad pública de la $\mathrm{Cd}$. de Chihuahua. La propuesta utilizó diversas técnicas: dinámica de grupos para la activación y desarrollo, lluvia de ideas, juegos de comunicación efectiva, cooperación, dramatización, dibujo, grupos auto dirigidos y técnicas de resolución de conflictos en equipo, así mismo, abordó otras técnicas de estimulación para un afrontamiento efectivo, en lo cognitivo conductual y estrategias fisio-afectivas, mediante foros de discusión y debate, en la regulación de temores colectivos dentro del contexto violento, para la solución de problemas reales.

Tabla 1

Esquema del Tipo de Diseño de la intervención

\begin{tabular}{ccccc}
\hline Grupos & Asignación & & \multicolumn{2}{c}{ Secuencia de registro } \\
\hline $\begin{array}{c}\text { Grupo } \\
\text { Tratamiento } \\
(G T)=26 \text { sujetos }\end{array}$ & $N A$ & Pre- Test & Tratamiento & Post-Test \\
& $\begin{array}{c}O_{t 1} \\
\text { (índice de } \\
\text { riesgo) }\end{array}$ & $X$ (programa) & $\begin{array}{c}O_{t 2} \\
\text { (índice de } \\
\text { riesgo) }\end{array}$ \\
$\begin{array}{c}\text { Grupo Control } \\
\text { (GC)=26 } \\
\text { sujetos }\end{array}$ & $N A$ & - & - & $\begin{array}{c}O_{c 2} \\
\text { (índice de } \\
\text { riesgo) }\end{array}$ \\
\end{tabular}

Nota: $N A=$ Asignación No Aleatoria, $O_{t l}=$ grupo tratamiento pre-test, $O_{t 2}=$ grupo tratamiento post-test, $O_{c 2}=$ grupo control post test,$X=$ tratamiento. Fuente: elaboración propia.

\section{Diseño}

Se trató de un estudio transversal, ya que, recuperó los datos en un momento determinado, con cierta población específica, estudiando las mismas variables, para todos los sujetos. Se realizó una entrevista inicial, se determinaron los perfiles socio-demográficos, de exposición a la violencia, clasificándose en función del: nivel de exposición a episodios de delito social, en: bajo, medio, alto. Según sustento teórico de clasificación para delitos de orden social, y de manera cualitativa, mediante una entrevista inicial en un 
formato de auto informe, semi-estructurada, previa a la aplicación de la batería de escalas cuantitativas.

\section{Participantes}

La muestra estuvo constituida por 26 adolescentes de 17 a 21 años, 1 grupo tratamiento (de 26 personas) y 1 grupo control (de 26 personas), 52 participantes, de los cuales $90.38 \%$ fueron mujeres y $9.62 \%$ hombres, jovenes que residen en Chihuahua, Chih, México.

\section{Procedimiento}

El programa de Intervención se desarrolló en 11 sesiones didácticas, un total de 44 horas de taller para la prevención de violencia, con alumnos de enseñanza superior pública de la ciudad de Chihuahua, Chihuahua, México.

Se comparó un grupo tratamiento, compuesto por: jóvenes de 17 a 21 años, de educación pública superior. Se analizó en una primera fase, las diferencias intra sujetos del tratamiento en el primer y segundo tiempo, pre y post, y en una segunda fase, se analizan las diferencias entre sujetos tratamiento y grupo control, en el segundo tiempo (post).

El taller estuvo constituido por actividades distribuidas en siete módulos, incluyeron de 8 a 10 actividades enfocadas a: erradicar la percepción de vulnerabilidad y distorsiones cognitivas, miedo social (concreto y difuso), e incremento de la eficacia colectiva, promotor de un afrontamiento cognitivo conductual proactivo, en colectivos infiltrados por la violencia social. Dichos módulos fueron: 1) vulnerabilidades y capacidades, 2) regulación físico emocional, 3) afrontamiento cognitivo conductual proactivo, 4) expresión, comprensión y manejo de miedos colectivos, 5) manejo de episodios de enojo, tristeza y alegría, 6) relaciones de cooperación y participación ciudadana, 7) propuesta de desarrollo comunitario mi vecindario saludable.

Se aprovechó para indagar sobre los diferentes tipos de miedo social (miedo concreto y miedo difuso); Así como, las estrategias de afrontamiento que utilizan los jóvenes, para ofrecer pautas o estrategias de acción, a manera de prevenir el escalamiento del nivel actual de violencia social, temáticas que regularmente no se trabajan en los créditos que se ofrecen de manera obligatoria, en el sistema educativo.

El grupo control, se conformó con muestra de la misma localidad, con historia y experiencias similares, a las del grupo tratamiento. Dicho grupo sin tratamiento, fue el grupo control no equivalente, en tanto los grupos tratamiento del programa de intervención en violencia $(X)$, fueron intervenidos a través de: el aprendizaje social de habilidades auto-reguladoras (guías para afrontamiento positivo, erradicación de miedo social y modificador de la conducta agresiva para una finalidad prosocial (de reactiva a proactiva), evitando el desgaste emocional en las relaciones sociales entre pares, en 
eficiencia colectiva, cultura ciudadana, respeto de normas y participación ciudadana, así mismo, participaron masivamente, en el proyecto de desarrollo: "vecindarios saludables", promoviendo la solución de necesidades en cada uno de sus entornos comunitarios inmediatos.

\section{Instrumentos}

Para la toma de medidas, se utilizó una batería de escalas de auto informe, que mide las variables de investigación, y para el desarrollo del curso y respaldo cualitativo, se realizaron entrevistas a profundidad respecto a victimización, grupos de discusión, exposiciones y resolución de casos grupales, finalizando con un proyecto de creación "mi vecindario saludable".

El factor principal del estudio denominado como: estrategias de afrontamiento, fueron medidas a través del: Cuestionario de Seguridad Urbana (CIU) desarrollado por la Dra. Roxana Vuanello, de la Universidad Nacional de San Luis, República Argentina, en el 2006. Esta Escala, está compuesta por cuatro factores de estilo de afrontamiento: afectivo y fisiológico, procesamiento cognitivo de negación, procesamiento cognitivo de afrontamiento y promoción conductual (Vuanello, 2006). Su aplicación en San Luis, Argentina, reportó en dicho estudio un alfa de cronbach de 0.82. En la prueba piloto se obtuvo una consistencia interna de 0.83 , lo cual nos indica, que resulta confiable.

La escala de miedo concreto (riesgo percibido a la victimización) y la escala de miedo difuso desarrolladas ambas por Ruiz, J.I. (2007), y La Escala de Cultura Ciudadana desarrollada por Ruiz, J.I. (2007) fueron aplicadas a los alumnos bajo tratamiento. Es importante destacar, que se elaboró una bitácora personal de cada uno de los jóvenes, una entrevista semi-estructurada para medir el nivel y tipo de victimización, para su clasificación en las dinámicas de las diversas sesiones grupales, a manera de obtener grupos víctimas y no víctimas en cada una de las sesiones de la intervención, de manera explícita y guiada a los participantes que indicaran los tipos de delitos a los que han estado expuestos en lo personal, familiar o de ser presenciados por un conocido, narrando situaciones complejas, donde se describían: percepciones, sentimientos y reacciones fisiológicas ante las situaciones criminales, de manera explícita, con la finalidad de detectar el tipo de victimización que presentaban; Así mismo, para obtener una referencia previa a la aplicación de la batería de escalas, un indicador que brindara mayor explicación de las vulnerabilidades de la muestra y sus procesos de afrontamiento.

\section{La Escala de miedo concreto (pre-post):}

Escala de Miedo Concreto (riesgo percibido). Consiste en una lista de 17 delitos, sub-escala desarrollada y adaptada de Peña (2005), y se pide a cada 
sujeto que indique si consideraba: poco probable (1), probable (2) o muy probable (3) que le ocurriera cada uno de los delitos, a futuro, en específico la probabilidad de ser víctima directa o indirecta, en los siguientes doce meses, Se incluyen delitos, con severidades entre, leves, moderadas y altas, desde: robo del carro, de la vivienda, robo a mano armada, hasta: secuestro, persecución por desconocidos, desaparición $u$ homicidio. En una investigación, el $\alpha$ de cronbach de la escala fue de 0.90 (Peña, 2005). De la suma de las respuestas a cada uno de los ítems y dividiendo el resultado entre el número de ítems se obtiene un puntaje en miedo concreto o percepción de riesgo ante el crimen, en el que, a mayor puntaje, mayor riesgo percibido de sufrir un delito en el futuro, mide 17 ítems, incluye delitos frecuentes a nivel internacional. Finalmente, (Ruiz, 2007), incluyó dos conductas: ser perseguido por desconocidos y recibir llamadas anónimas obscenas, que no suelen ser tipificadas como delitos, debido a que anteriormente, no habían mostrado asociación a la percepción de riesgo. El coeficiente alfa de cronbach, de fiabilidad interna de la escala, al aplicarse en Bogotá, Colombia, en el año 2001, fue de 0.87 (Ruiz, 2007). Al realizar nuestro análisis en Chihuahua, Chih, México, se obtiene un alfa de 0.96 , lo que significa, que la escala es altamente confiable.

La Escala de miedo difuso (pre-post): Esta Sub-escala consiste en una lista que mide 7 ítems, elaborada por Ruiz (2010), ítems como: miedo a ser víctima de un delito y el miedo a ser victimizado en el hogar. Los ítems en formato Likert, con cuatro opciones de respuesta, desde: 1 (nada) hasta 4 (mucho). Es una sub-escala, que resulta de la combinación de un grupo de tres ítems sobre miedo difuso de Ruiz (2004), adaptada con dos ítems de la Encuesta Internacional de Victimización, más otro ítem sobre temor dentro del hogar. Estos tres ítems, tuvieron un alfa de cronbach de 0.78 (Ruiz, 2004). Los otros tres ítems, se refieren al grado de temor respecto a la colonia, y al municipio (Peña, 2005). Esta escala, ha mostrado una alta consistencia interna ( alfa de Cronbach de 0.80), en trabajos posteriores, se obtuvo un alfa de cronbach de 0.76 , la escala fue confiable en un estudio piloto para la ciudad de Chihuahua, Chih. México.

La Escala de cultura ciudadana (pre-post): compuesta de ocho indicadores: compromiso por la ciudad, respeto por la ciudad, identificación afectiva, servicios de la ciudad, participación ciudadana, respeto por las normas sociales, civismo y tolerancia, así como intención de cuidado de los espacios públicos. El índice de confiabilidad interna de la escala, mostró un alfa de cronbach de 0.93, en una muestra aplicada en Bogotá, Colombia. (Ruiz, 2010). 


\section{Resultados}

\section{Primera Fase de Análisis}

Utilizando el programa estadístico SPSS ( Statistical Program for Social Sciences), se realizó el análisis de datos de nuestro estudio, dicho análisis consistió de dos fases: en primer lugar, se realizó una prueba "T" para muestras relacionadas inter sujetos, en los tiempos pre y post, del grupo tratamiento. En una segunda fase, se analizan las diferencias entre sujetos tratamiento y grupo control, en el segundo tiempo (post).

\section{Tabla 2}

Medias y desviaciones estándar (entre paréntesis) de los principales factores de riesgo y pro-sociales del grupo tratamiento, en los tiempos pre y post.

\begin{tabular}{cccccc}
\hline & \multicolumn{3}{c}{ Pre-Intervención } & \multicolumn{2}{c}{ Post-Intervención } \\
\hline Factor de Riesgo & $\boldsymbol{N}$ & Media $(\boldsymbol{\sigma})$ & Error & Media $(\boldsymbol{\sigma})$ & Error \\
Miedo Concreto & 26 & $97.81(23.03)$ & 4.52 & $73.38(11.19)$ & 2.20 \\
Miedo Difuso & 26 & $18.42(4.21)$ & 0.82 & $13.08(3.33)$ & 0.65 \\
Afrontamiento Afectivo & 26 & $86.24(40.13)$ & 8.03 & $35.60(25.13)$ & 5.03 \\
Afrontamiento & 20 & $52.92(38.58)$ & 7.72 & $20.62(18.57)$ & 3.71 \\
$\quad$ Fisiológico & & & & & \\
\hline Factor Pro Social & 26 & $90.50(15.64)$ & 3.07 & $106.96(16.55)$ & 3.25 \\
Cultura Ciudadana & 26
\end{tabular}

Nota: Miedo Concreto= percepción de riesgo de los jóvenes, Miedo Difuso= Miedo emocional al crimen, Afrontamiento Afectivo= rasgos de afrontamiento emocional de la delincuencia en los jóvenes; Afrontamiento Fisiológico= rasgos de somatización física de la violencia en los jóvenes, Cultura Ciudadana= aspectos de participación cívica, afecto por la ciudad, respeto a las normas y a los ciudadanos. Fuente: elaboración propia.

El grupo tratamiento, muestra cambios estadísticamente significativos en puntuaciones de medias al término de la intervención, a su vez, se analiza el tamaño de efecto de tratamientos, según sustento metodológico, de Cohen (1992).

La escala de cultura ciudadana, que refiere aspectos de cultura cívica, en: participación ciudadana, afecto por la ciudad, respeto a normas y ciudadanos, presentó variación de puntuaciones entre pre y post test del tratamiento.

El factor de riesgo percibido, a nivel personal, presenta una diferencia de puntuaciones en las medias estadísticamente significativa, de: $\bar{X}=11.46$, con un tamaño de efecto, determinado por $\mathrm{d}$ de Cohen $=2.93$, entre pre y post test, mientras que: el riesgo percibido familiar muestra una diferencia de medias de: $\bar{X}=11.92$ y d de Cohen $=4.17$; es decir, magnitudes de efecto grandes.

La percepción de riesgo respecto a percepción de vulnerabilidad de un conocido, presenta una diferencia de puntuación en medias de: $\bar{X}=10.95$ y $\mathrm{d}$ de Cohen $=3.89$; la percepción de riesgo a ser victimizado (miedo concreto) presenta una diferencia de medias de: $\bar{X}=24.43$ y d de Cohen $=3.19$; un tamaño de efecto grande, la escala de cultura ciudadana, que implica la 
participación ciudadana, entre otros factores, presenta diferencia de medias de: $\bar{X}=-16.50$ y d de Cohen $=-2.29$, un incremento grande, el miedo difuso, presenta una diferencia de medias de: $\bar{X}=5.34$ y d de Cohen $=3.17$; La estrategia de afrontamiento afectivo presenta una diferencia de medias de: $\bar{X}$ $=50.64 \mathrm{y} \mathrm{d}$ de Cohen= 3.49, efectos de magnitud grande .

La estrategia de afrontamiento cognitiva, muestra una diferencia de medias de: $\bar{X}=29.86$ y d de Cohen= 1.86 ; La estrategia de afrontamiento fisiológico muestra una diferencia de medias de: $\bar{X}=32.32 \mathrm{y}$ d de Cohen= 2.53 ; es decir, un efecto grande.

Respecto a los resultados obtenidos, se puede concluir que: se rechaza la Hol (Hipótesis nula) pues existen diferencias en las puntuaciones de las medias, estadísticamente significativas entre el pre y post test (intra sujetos). $\mathrm{Al}$ retomar la primera Hipótesis de Investigación $\left(H i_{1}\right)$, el planteamiento fue el siguiente: Existen diferencias significativas en los niveles de riesgo inherentes a estrategias de afrontamiento afectivas, cognitivas, fisiológicas y conductuales; a nivel personal, familiar y comunitario, en torno al miedo emocional al crimen o miedo difuso y la percepción de riesgo intra sujetos después de la intervención (grupo tratamiento pre y post).

Aunado a esto, se observan tamaños de efecto considerablemente grandes en todos los casos, el tamaño de efecto en todos los factores, que resultaron mayores que 0.80; es decir, la magnitud de la diferencia intra sujetos tratamiento en el primer y segundo tiempo es alto.

\section{Tabla 3}

Prueba "t" para muestras relacionadas y resultados de las puntuaciones obtenidas en las escalas en el Pre y Post, del grupo tratamiento.

\begin{tabular}{cc}
\hline Escala & Resultado Prueba T \\
\hline Miedo Concreto & $\mathrm{t}(25)=7.33, \mathrm{p}=.000$ \\
Miedo Difuso & $\mathrm{t}(25)=5.85, \mathrm{p}=.000$ \\
Afrontamiento Afectivo & $\mathrm{t}(24)=5.91, \mathrm{p}=.000$ \\
Afrontamiento Fisiológico & $\mathrm{t}(24)=4.27, \mathrm{p}=.000$ \\
Cultura Ciudadana & $\mathrm{t}(25)=3.85, \mathrm{p}=.001$ \\
\hline
\end{tabular}

Nota: Miedo Concreto=percepción de riesgo de los jóvenes, Miedo Difuso $=$ Miedo emocional al crimen, Afrontamiento Afectivo= rasgos de afrontamiento emocional de la delincuencia en los jóvenes, Afrontamiento Fisiológico= rasgos de somatización física de la violencia en los jóvenes, Afrontamiento Cognitivo: estrategias y cogniciones para afrontar la violencia, Cultura Ciudadana = aspectos de participación cívica, de respeto y afecto por la ciudad, a las normas y a sus ciudadanos. Fuente: elaboración propia.

Después de realizar la Prueba $T$ para muestras relacionadas, se identificaron diferencias en las puntuaciones pre y post pruebas estadísticamente significativas, y se rechaza la $\mathrm{Hol}$ (hipótesis nula) en las variables principales, Para el miedo concreto total $t(25)=7.33, p=.000$, cultura ciudadana $t(25)=-3.86, \mathrm{p}=.001$, miedo difuso total $t(25)=5.86, p=.000$, estrategia de afrontamiento afectivo $t(24)=5.91, p=.000$, estrategias de 
afrontamiento fisiológicas $t(24)=4.27, p=.000$, total de miedo social $t(25)=7.26, p=.000$.

En efecto, el tratamiento muestra cambios en las puntuaciones de las medias respecto a los factores de riesgo, en los procesamientos cognitivos y conductuales negativas o antisociales y en respuestas afectivo fisiológicas, que en grados extremos denotan algunos rasgos de estrés postraumático en las poblaciones juveniles; así como, una variación estadísticamente significativa de las puntuaciones en las medias en la cultura y participación ciudadana del grupo que estuvo bajo tratamiento.

\section{Segunda Fase de Análisis}

Se trabajó con un grupo control de $N=26$ jóvenes en el post test, los resultados del mismo se compararon con aquellos obtenidos con el grupo tratamiento en el post test. Los resultados de los grupos control y tratamiento se analizan a continuación, mediante Anova de un factor, se presentan los estadísticos descriptivos del análisis:

Tabla 4. Medias y desviaciones típicas (entre paréntesis) de los principales factores pro sociales (+) y factores de riesgo(-)trabajados en la intervención, comparativo entre grupo tratamiento y grupo control en el segundo tiempo (post)

\begin{tabular}{|c|c|c|c|c|}
\hline Factor $(+,-)$ & $\mathrm{g}$ & $\mathrm{N}$ & Media $(\sigma)$ & Error típico \\
\hline Miedo concreto(-) & $\begin{array}{l}\mathrm{t} \\
\mathrm{c}\end{array}$ & $\begin{array}{l}26 \\
26 \\
52\end{array}$ & $\begin{array}{l}73.38(11.19) \\
82.65(23.05) \\
78.01(18.54)\end{array}$ & $\begin{array}{l}2.19 \\
4.52 \\
2.57\end{array}$ \\
\hline Cultura ciudadana $(+)$ & $\begin{array}{l}\mathrm{t} \\
\mathrm{c}\end{array}$ & $\begin{array}{l}26 \\
26 \\
52\end{array}$ & $\begin{array}{c}106.96(16.55) \\
88.73(15.33) \\
97.84(18.28)\end{array}$ & $\begin{array}{l}3.24 \\
3.00 \\
2.53\end{array}$ \\
\hline Miedo difuso( - ) & $\begin{array}{l}\mathrm{t} \\
\mathrm{c}\end{array}$ & $\begin{array}{l}26 \\
26 \\
52\end{array}$ & $\begin{array}{l}13.07(3.33) \\
17.42(4.26) \\
15.25(4.37)\end{array}$ & $\begin{array}{l}0.65 \\
0.83 \\
0.60\end{array}$ \\
\hline Afrontamiento afectivo( - ) & $\begin{array}{l}\mathrm{t} \\
\mathrm{c}\end{array}$ & $\begin{array}{l}26 \\
26 \\
52\end{array}$ & $\begin{array}{l}35.23(24.69) \\
91.03(40.74) \\
63.13(43.65)\end{array}$ & $\begin{array}{l}4.84 \\
7.98 \\
6.05\end{array}$ \\
\hline Afrontamiento fisiológico( - ) & $\begin{array}{l}\mathrm{t} \\
\mathrm{c}\end{array}$ & $\begin{array}{l}26 \\
26 \\
52\end{array}$ & $\begin{array}{l}21.07(18.35) \\
66.73(50.04) \\
43.90(43.86)\end{array}$ & $\begin{array}{l}3.60 \\
9.81 \\
6.08\end{array}$ \\
\hline
\end{tabular}

\footnotetext{
Nota: factor $+=$ factor pro social, factor $-=$ factor de riesgo, $g=$ grupo, $t=$ tratamiento, $c=$ control, Miedo Concreto=percepción de riesgo de los jóvenes; Miedo Difuso= Miedo emocional al crimen; Afrontamiento Afectivo= rasgos de afrontamiento emocional de la delincuencia en los jóvenes, Afrontamiento Fisiológico= rasgos de somatización física de la violencia en los jóvenes; Cultura Ciudadana $=$ aspectos de participación cívica, afecto por la ciudad, respeto a las normas y a los ciudadanos. Fuente: elaboración propia
} 
Los jóvenes participantes en el grupo control, de características socio demográficas y etarias similares al grupo tratamiento, fueron seleccionados por conveniencia considerando que los horarios en que ellos cursaban, no lograron acomodarse a los de aquellos que participaron en el taller intervención, dicha muestra fue recolectada en la misma área geográfica, en el mismo centro de estudios, y con sujetos bajo las mismas condiciones, a los cuales se les aplicó la batería de escalas que fue utilizada con los sujetos tratamiento, en el segundo tiempo (post test).

Tabla 5. Anova de un factor grupo tratamiento y grupo control (post)

\begin{tabular}{ccc}
\hline Escala & Resultado Anova & d de Cohen \\
\hline Miedo Concreto & $\mathrm{F}(1,50)=3.40, \mathrm{p}=.071 \quad$ no significativa \\
Cultura Ciudadana & $\mathrm{F}(1,50)=16.97, \mathrm{p}=.000 \mathrm{~d}=1.14$ \\
Miedo Difuso & $\mathrm{F}(1,50)=16.76, \mathrm{p}=.000 \mathrm{~d}=1.14$ \\
Afrontamiento Afectivo & $\mathrm{F}(1,50)=35.69, \mathrm{p}=.000 \mathrm{~d}=1.71$ \\
Afrontamiento Fisiológico & $\mathrm{F}(1,50)=19.07, \mathrm{p}=.000 \mathrm{~d}=1.33$ \\
Afrontamiento Cognitivo Negativo & $\mathrm{F}(1,50)=10.18, \mathrm{p}=.002 \quad \mathrm{~d}=0.89$
\end{tabular}

Nota: Miedo Concreto=percepción de riesgo de los jóvenes, Miedo Difuso= Miedo emocional al crimen, Afrontamiento Afectivo= rasgos de afrontamiento emocional de la delincuencia en los jóvenes, Afrontamiento Fisiológico= rasgos de somatización física de la violencia en los jóvenes, Cultura Ciudadana= aspectos de participación cívica, afecto por la ciudad, respeto a las normas y a los ciudadanos, Afrontamiento Cognitivo Negativo= pensamientos o cogniciones antisociales, $\mathrm{d}$ de Cohen= determina el tamaño de efecto estadístico, análisis de poder o magnitud del cambio en las variables intra / inter sujetos, al detectarse diferencias estadísticamente significativas. Fuente: elaboración propia

El riesgo percibido personal $F(1,50)=3.99, p=.05, d=0.55$, muestra diferencias estadísticamente significativas entre tratamiento y control, con tamaño de efecto moderado, de acuerdo a Cohen (1992), los efectos de 0.20 son bajos, de 0.50 son moderados y los efectos de 0.80 son altos. El riesgo percibido familiar $F(1,50)=14.97, p<.001, d=1.13$, muestra diferencias significativas entre grupo tratamiento y control, con un tamaño de efecto grande; El riesgo percibido de que un conocido sea victimizado muestra una $F(1,50)=12.72, p=.001, d=1.04$, el miedo concreto $F(1,50)=3.40, p=.07$, no muestra diferencias significativas en grupo tratamiento y control.

En el caso del factor cultura ciudadana, encontramos una $\mathrm{F}$ $(1,50)=16.97, p<.001, d=1.14$, muestra variación significativa entre grupo tratamiento y control, con tamaño de efecto grande.

Respecto al miedo difuso, el temor emocional al crimen muestra $F$ $(1,50)=16.76, \quad p<.001, \quad d=1.14 ; \quad$ muestra diferencias estadísticamente significativas entre grupo tratamiento y control, con un tamaño de efecto alto.

Analizando las estrategias de afrontamiento se muestran cambios en puntajes de medias estadísticamente significativos en las respuestas de afrontamiento afectivas $F(1,50)=35.7, p<.001, d=1.71$, entre tratamiento y grupo control, en lo referente a: las estrategias cognitivas negativas 
(mecanismos de negación y restricción cognitiva) $F(1,50)=10.18, p=.002$, $d=0.89$, estrategias o respuestas fisiológicas $F(1,50)=19.07, p<.001, d=$ 1.33 , estrategias conductuales antisociales, con una $F(1,50)=9.86, p=.003$, $d=0.87$, todas las anteriores muestran diferencias de puntajes de medias entre pre y post prueba estadísticamente significativos.

Nuestra segunda hipótesis de investigación plantea: Hi2: Existen diferencias significativas en las puntuaciones de las medias en las estrategias de afrontamiento afectivas, fisiológicas, cognitivas y conductuales: a nivel personal, familiar y comunitario, en torno al miedo emocional al crimen o miedo difuso y la percepción de riesgo entre el grupo tratamiento y los jóvenes del grupo control, se puede concluir que se rechaza la Ho2 (Hipótesis nula) pues existen diferencias estadísticamente significativas entre sujetos tratamiento y control.

Estos hallazgos y los anteriormente citados, no implican una presencia de causalidad solamente implican una diferencia en puntajes de las medias, debido a que es un diseño cuasi experimental y las unidades no fueron asignadas de manera estrictamente aleatoria a los grupos.

\section{Discusión}

Antes de reportar los hallazgos del tratamiento, es importante destacar que en un estudio cuasi experimental, por cuestiones de logística las unidades de investigación no resultaron asignadas de manera aleatoria, y que a pesar de que se manejo una planificación cuidadosa para familiarizarse con el contexto, en la aplicación y control de la intervención, la presunción de causalidad no es posible, además de que son múltiples factores los implicados en un estudio de corte psicosocial, y no todos son abordados durante el presente estudio, como podría ser el proceso de polarización grupal, que ocurre en la toma de decisiones grupal, cuando las personas se acercan al proceso sintiendo que sus opiniones son mejores que las de los demás miembros. Al gestarse la discusión del problema, los miembros comienzan a aprender que sus puntos de vista son cercanos al promedio, y no únicos, como respuesta, el individuo tiende a moverse a una posición más extrema, esto podría haberse controlado al existir un grupo tratamiento adicional que alternadamente tomara el taller, asignado de manera aleatoria.

La evaluación más rigurosa del modelo ampliado y perfeccionado que se recomienda involucraría el posible uso de un diseño controlado aleatorio, con un número mayor de participantes y de grupos tratamiento y control, durante un periodo de tiempo más largo. El estudio actual nos proporcionó datos con alentadores indicios que ameritan una mayor implementación y evaluación del modelo.

Su efecto en la cobertura de la necesidad, al fomentar el afrontamiento eficaz y atenuar el miedo colectivo, incrementando la pro actividad ciudadana, 
resulta prometedor e interesante para seguirse estudiando, esto a partir de los tamaños de efecto de carácter alto encontrados en el grupo tratamiento al comparar el primer tiempo respecto al segundo tiempo de la medición.

El tratamiento muestra diferencias estadísticamente significativas, en: las capacidades pro sociales de las y los adolescentes, por ende, resulta importante un seguimiento en la adaptación de los recursos aprendidos durante las sesiones y en los proyectos de mejora realizados al final del taller, denominados: "vecindarios saludables", con la finalidad de que el grupo tratamiento replique lo aprendido a pares inmediatos y a sus comunidades (efecto bola de nieve).

La mejora de la relación de las y los jovenes con otras personas de la comunidad y la posibilidad de que la intervención pueda provocar cambios ulteriores, es un punto a destacar.

Consideramos importante destacar algunas acotaciones de relevancia respecto al tamaño de efecto, de los tratamientos o intervenciones. Para Cohen (1992), entre más grande sea el tamaño del efecto en la población de estudio, más probable resulta que el efecto observado en el estudio alcance el criterio de significación estadística, y mayor será la potencia, el tamaño del efecto no puede variarse, se trata del fenómeno que se estudia; sin embargo, "la única estrategia posible resulta en el empleo de un suficiente número de observaciones, aunque este procedimiento resulte adecuado presenta el inconveniente de la dificultad para recoger una muestra amplia, por cuestiones de economía" (Cohen, 1992, p.157), la necesidad de garantizar los resultados de la investigación consiste en asumir los menores márgenes posibles de error de Tipo I , y a la vez de Tipo II . Para Cohen (1992) los efectos de 0.20 son bajos, de 0.50 son moderados y los efectos de 0.80 son altos o grandes, en el presente estudio se obtuvieron tamaños de efectos grandes, de suficiente significación estadística para rechazar la hipótesis nula. El tratamiento ofreció información efectiva y modificadora en pro de la cultura ciudadana, sin embargo, se requiere el ensayo en muestras más amplias, además de un diseño experimental más riguroso en el futuro, que permita establecer relaciones de causalidad.

Los resultados del primer estudio, los pre-test y post-test del grupo tratamiento se revisaron mediante prueba $T$ para medidas relacionadas para definir relaciones intra sujetos, existe variación significativa en factores de riesgo que afectan a la población en los procesamientos cognitivos y conductuales de connotación negativa y en respuestas afectivas y fisiológicas, que en grados extremos denotan rasgos de estrés postraumático, en las poblaciones juveniles.

La cultura y participación ciudadana del grupo, presentó variación entre el primer y segundo tiempo, para el grupo que estuvo durante 44 horas de taller tratamiento. 
A manera cualitativa, se obtuvieron hallazgos respecto a las relaciones inter-grupales, estrategias cognitivo conductuales de afrontamiento, mas asertivas en torno a situaciones sociales conflictivas, en capacidades para analizar sentimientos negativos, y en las estrategias tratadas, así mismo, se identifica que las conductas sociales de ansiedad y timidez, los problemas conductuales de timidez y retraimiento, así como: conductas antisociales fueron atenuándose durante el progreso del taller.

En síntesis, Se pretendía desde un inicio encontrar una reducción importante en la incidencia de procesamientos cognitivos de negación (mecanismos de defensa) al afrontar, conductas antisociales y violentas hacia el logro de un afrontamiento socioemocional positivo, así como, lograr atenuar los altos niveles de miedo social, reducción de afrontamiento afectivo y fisiológico con la pretensión de nivelar los rasgos de estrés postraumático, así mismo, se visualizó una mayor y mejor participación ciudadana, mediante propuestas de solución a las temáticas de los módulos, dentro de la perspectiva particular de las juventudes, los hallazgos indican cambios en estos factores.

Respecto a la segunda fase de análisis, comparando el segundo tiempo o post test tratamiento y control mediante Anova inter-sujetos, se obtiene reducción de puntuaciones medias significativas, con magnitud de efecto grande, entre el grupo tratamiento post test y control, a excepción del total de riesgo percibido personal, que pertenece a la escala de percepción de riesgo o miedo concreto, donde, se encontró que precisamente por la limitación de que el grupo fue prácticamente seleccionado por conveniencia, el grupo de manera inicial tenía un riesgo percibido personal mucho más bajo que el grupo tratamiento.

El resto de las variables estudiadas presentan diferencias de puntuaciones medias significativas, para 9 de las 11 variables estudiadas.

El número de personas beneficiadas por la intervención fue bajo, se contó con una muestra de 52 jovenes. La disponibilidad de recursos económicos, materiales y humanos para llevarlas a cabo, resultaron escasos, sin embargo, es factible conseguir diversos apoyos a nivel nacional a manera de gestionar un programa de intervención, inclusive la implementación de una materia o crédito en la currícula pública de la oferta académica que cursan los estudiantes, a manera de lograr la factibilidad del mismo con una cobertura más amplia.

El coste de la postergación del tratamiento del problema redunda en aun mayor vulnerabilidad, mayores disonancias y desajustes a propósito de su condición biológica y de habitar un entorno permeado de violencia; Sin embargo, al incidir en la resolución de algunos factores de riesgo que promueven aun mayor adversidad y desequilibrios, permitiríamos una evolución en dichos colectivos juveniles mediante determinados recursos 
personales de afrontamiento y resolución de esos desequilibrios identitarios, de seguridad y sociales, con un enfoque pro social y comunitario.

El programa diseñado para adolescentes de 17 a 21 años, presentó diferencias en las puntuaciones medias, estadísticamente significativas para un amplio abanico de factores del desarrollo socio-emocional, preventivos y de educación en cultura ciudadana y derechos humanos en jóvenes de contextos violentos.

Se observó un fomento en conductas de auto cuidado y protección ante la vulnerabilidad del contexto; Aún así, estar sometidos para la amenaza constante; aunado a enfrentarles con estrategias fisio afectivas deriva en mucho desgaste en las relaciones, una baja de la tolerancia social y disminución de la eficacia colectiva, aunado a esto, una alta percepción de riesgo en la esfera personal, familiar y comunitaria, pareció relacionarse a la presencia de rasgos de estrés postraumático en colectivos sometidos a violencia social, vulnerando su calidad de vida, conectividad y pro sociabilidad.

\section{References:}

1. Cohen, J. (1992). A Power Primer. Psychological Bulletin, 112, 155159.

2. Dammert, L. \& Malone, M.F. (2006). Does it take a village? Policing strategies and fear of crime in Latin America. Latin America Politics \& Society. 48 (4), 27-51.

3. Feixa, C. (1998). De jóvenes, bandas y tribus. Antropología de la juventud. Barcelona: Ariel

4. Gómez, C.E. (2008). Adolescencia y familia: revisión de la revisión y la relación y la comunicación como factores de riesgo o protección. Revista Intercontinental de Psicología y Educación, 10 (2), 105-122

5. Lanford, J., Dodge, K., Dodge, K., Bates, J. \&Petit, G. (2002). Developmental trajectories of reactive and proactive aggression. Paper presented at the 15th World Meeting of the International Society for Research on Aggression, Montreal, p. 109.

6. Niño, S., Lugo, N., Rozo, C. \&Vega, L. (1998). Territorios del miedo en Santafé de Bogotá: imaginarios de los ciudadanos. Bogotá: TM Editores y Observatorio de Cultura Urbana.

7. Peña, M. (2005). Conducta antisocial. Factores de riesgo y de protección. Tesis doctoral. Universidad Complutense de Madrid.

8. Ruiz, J.I. (2004). Un modelo sociocultural del encarcelamiento: afectividad, factores psicosociales y cultura. San Sebastián: Universidad del País Vasco. Tesis de doctorado.

9. Ruiz, J.I. (2005). Cultura ciudadana: sus dimensiones psicosociales. Cuadernos Hispanoamericanos de Psicología. 5 (1), 59-76. 
10. Ruiz, J.I. (2007). Cultura ciudadana, miedo al crimen y victimización: un análisis de sus interrelaciones desde la perspectiva del tejido social. Acta Colombiana de Psicología.

11. Ruiz, J.I. (2010). Eficacia Colectiva, Cultura Ciudadana y Victimización. Acta Colombiana de Psicología, Bogotá, Colombia.13(1) , 26-29.

12. Santos, J.I., Villa, M.J.P., García, A., León, G., Quezada, S. \&Tapia, R. (2003). La transición epidemiológica de las y los adolescentes en México. Salud pública en México, 45 (1), 140-152.

13. Villareal, A.\& Silva, B.F.A. (2006). Social cohesion, criminal victimization and perceived risk of crime in Brazilian neighborhoods. Social Forces, 84 (3), 1725-1753.

14. Vozmediano, L. \& San Juán, C. (2006). Empleo de Sistemas de Información Geográfica en el estudio del Miedo al Delito. Revista Española de Investigación Criminológica. 2 www.criminologia.net. (recuperado el 26 de octubre del 2014).

15. Vuanello, R. (2006). Un nuevo instrumento de Evaluación Psicológica: El Cuestionario de Inseguridad Urbana. Interdisciplinaria, 23, 1:17-45. 